\title{
EDITORIAL
}

\section{Anaplastic large cell lymphoma: the current state of play from a European prospective as presented at the second annual meeting of the European Research Initiative on ALCL, 27-28 June 2011}

Leukemia (2011) 25, 1795-1796; doi:10.1038/leu.2011.295

The European Research Initiative on Anaplastic Large Cell Lymphoma (ERIA) was conceived in Denver, CO, USA at the 100th Annual Meeting of the American Association for Cancer Research when three scientists (Drs Suzanne Turner, Olaf Merkel and Lukas Kenner) with a common interest met at a poster session. The rest, as they say is history, but following a second successful meeting we are certain there is history yet to be made.

The idea at the poster session has now blossomed into a study group that has members from at least five European countries, including Austria, France, Germany, Italy and the United Kingdom and in the last year has expanded to involve our clinical colleagues. Our members now cross many specialities from diagnostic histopathologists to laboratory scientists and practicing clinicians. Through this initiative we intend to develop collaborations and strengthen cooperations between research groups in Europe, all with a common aim of furthering our understanding of anaplastic large cell lymphoma (ALCL) with the long-term goal of developing less toxic and more efficacious therapies for those affected patients.

The 'Inaugural Meeting of the European ALCL Study Group' was held in Salzburg, Austria in June 2010. Our keynote speaker was Giorgio Inghirami who presented a thorough and all-encompassing history of ALCL and the important role that the transcription factor STAT3 has in the propagation of survival signals in this disease. Other speakers included Justus Duyster, Suzanne Turner, Olaf Merkel, Lukas Kenner, Emanuela Colombo, Stephan Mathas, Sylvie Giuriato, Roberto Chiarle and Karen Pulford. The success and enthusiasm of this meeting has started what we hope will become a tradition with the second meeting held a year later in Vienna, Austria.

The theme of our second annual meeting was twofold, first to examine emerging themes in this specialised area of research and second to explore current therapies and future clinical trials. Our plenary lecture was presented by Dr Karen Pulford of the University of Oxford, UK who spoke from the Oxford perspective of the emergence of $\mathrm{ALCL}$, anaplastic lymphoma kinase $(\mathrm{ALK})^{+}$as a diagnostic entity acknowledging contributions made by the late Prof David Mason. Karen also presented recent data detailing the outcome of a fruitful collaboration with Alfred Reiter, Willi Woessmann and Christine Damm-Welk (Giessen, Germany) in which the role of preexisting ALK antibodies at the time of ALCL diagnosis was studied. It was demonstrated that high titres of ALK antibodies correlate with low numbers of circulating tumour cells and more importantly lower cumulative incidence of relapse. This gives experimental support for the concept of immune-surveillance in $\mathrm{ALCL}, \mathrm{ALK}^{+}$.

\section{The clinical perspective}

We were fortunate to receive presentations from two respected clinicians; one focused on adult lymphoma and the other presented from the paediatric angle. Prof Norbert Schmitz representing the German high-grade lymphoma study group (http://www.lymphome.de/en/Groups/DSHNHL) presented the German perspective of adult T-cell lymphomas of which ALCL, specifically $\mathrm{ALK}^{+}$cases, have the best overall survival. In adults, the average age of presentation with $\mathrm{ALCL}, \mathrm{ALK}^{+}$is 37 years whereas this is considerably higher at 50 years for the $\mathrm{ALK}^{-}$ cases. Current therapy is in the form of the archetypal CHOP regimen and at present there are no plans to replace this, only to supplement it with novel agents such as the armed CD30 antibody SGN35.

Similarly, ALCL presenting in paediatric patients has a good prognosis with overall survival rates reaching $90 \%$. Dr Willi Woessmann presented data from the ALCL 99 clinical trial, which served to evaluate the efficacy of vinblastine when added to the short pulse chemotherapy received by children. This study enrolled patients from 15 countries, but did not show any advantage to vinblastine treatment except for a slight delay in time to relapse for some patients. Interestingly, those patients that relapse have an overall survival rate of $57-70 \%$ and so despite the relative success of initial treatment, we still have some way to go. To this end Willi presented data showing that although clinical risk factors do not predict high-risk patients, the PCR of bone marrow ALK levels does (greater than 10 copies is associated with an $80 \%$ event-free survival (EFS) whereas less than 10 copies indicates $30 \% \mathrm{EFS}$ ) as do the peripheral blood ALK antibody titres (a titre greater than $1 / 750$ is associated with $78 \%$ EFS and those below this value with a $40 \%$ EFS). Any future trials will most likely involve a risk stratification step assessing ALK levels in the bone marrow and ALK antibody titres. What is clear is that any future trials are dependent on Europe if not worldwide co-operation to enrol sufficient patients.

\section{Emerging themes}

A variety of lab-based science projects were presented at the meeting; it became clear that there are some common emerging themes including the role of micro (mi)RNAs in the pathogenesis of ALCL. miRNAs are small RNAs of 18-22 nucleotides that regulate protein expression by semi-perfect base pairing mostly to the $3^{\prime}$-UTR of their target genes. In the last decade they have gained a reputation as important players in oncogenesis by regulating processes as diverse as apoptosis, senescence, the immune response and haematopoiesis. In the Olaf Merkel group (Salzburg, Austria) miRNA expression in ALCL was studied in cell lines, a murine model as well as in primary patient tissue. It was demonstrated that 
$\mathrm{ALK}^{+}$and $\mathrm{ALK}^{-} \mathrm{ALCL}$ can be distinguished based on their miRNA expression profile. In particular, high expression of the oncogenic miR17-92 cluster is typical for $\mathrm{ALK}^{+} \mathrm{ALCL}$, whereas the oncogenic miR-155 is higher in $\mathrm{ALK}^{-} \mathrm{ALCL}$. Dr Laurence Lamant (Toulouse, France) spoke about another miRNA that seems to be epigenetically repressed by the ALK kinase, miR-29a. This repression may explain the high level of the prosurvival protein $\mathrm{Mcl}-1$ in $\mathrm{ALK}^{+} \mathrm{ALCL}$. Finally, they showed that miR-29 restoration in the $\mathrm{ALK}^{+} \mathrm{ALCL}$ cell line Karpas 299 results in reduced tumour growth when engrafted in non-obese diabetic-severe combined immunodeficient mice.

Lukas Kenner presented data regarding the role of AP-1 transcription factors in the progression of ALCL. In his lab it was shown that knockout of Jun proteins in a murine nucleophosmin (NPM)-ALK-driven transgenic tumour model led to a change in the aggressiveness of the ensuing lymphoma; organ infiltration was reduced when Jun proteins were absent. Furthermore, he presented unpublished data regarding therapeutically interesting targets of AP-1 in ALCL. This work was performed in collaboration with other ERIA members, namely Giorgio Inghirami and Olaf Merkel.

Other talks showed that mRNA metabolism is influenced by NPM-ALK expression (Estelle Espinos, Toulouse) and that cdk6 has an underestimated role in this disease (Veronika Sexl, Vienna). Leticia Quintanilla-Fend (Munich, Germany) also presented her data concerning the role of C/EPP $\beta$ in the pathogenesis of ALCL.

\section{Concluding remarks}

It is clear that there have been many developments in the field and that we are rapidly increasing our understanding of this disease and furthermore are translating these findings to the clinic. We are also excited about the renewed interest in the ALK oncogene following the identification of ALK deregulation in solid tumours including neuroblastoma and non-small cell lung carcinoma. We therefore are looking forward to our third annual meeting, which will take place in Cambridge, England on the 2-3 July 2012. Please visit our homepage for details (http://www.erialcl.net).

\section{Conflict of interest}

The authors declare no conflict of interest.

O Merkel ${ }^{1}, \mathrm{~L}$ Kenner ${ }^{2}$ and SD Turner $^{3}$

${ }^{1}$ Laboratory for Immunological and Molecular Cancer Research, 3rd Medical Department with Hematology, Medical Oncology, Hemostaseology, Rheumatology and Infectiology of the Private Medical University Hospital, Salzburg, Austria;

${ }^{2}$ Ludwig Boltzmann Institute for Cancer Research, and Institute for Clinical Pathology, Medical University Vienna, Vienna, Austria and

${ }^{3}$ Division of Molecular Histopathology, Department of Pathology, University of Cambridge, Addenbrooke's Hospital, Cambridge, UK

E-mail:sdt36@cam.ac.ukor lukas.kenner@meduniwein.ac.at oro.merkel@salk.at 